\title{
Loss of Imprinting of Insulin-Like Growth Factor 2 is Associated with Increased Risk of Primary Lung Cancer in the Central China Region
}

\author{
Ming Zhang', Cui-Huan $\mathrm{Wu}^{2}$, Xiao-Ling Zhu' ${ }^{3}$, You-Jie Wang ${ }^{3 *}$
}

\begin{abstract}
Background: To determine the imprinting status of the IGF2 in Chinese patients with primary lung cancer and to analyze the clinical significance of the loss of imprinting (LOI) of IGF2. Materials and Methods: PCRRFLP and RT-PCR-RFLP were carried out to select heterozygous cases for the ApaI polymorphism within exon 9 of the IGF2 gene and further analyze IGF2 LOI in 64 lung cancer patients, respectively. Results: Of 64 lung cancer patients, 31 were heterozygous for IGF2. The positive rates of IGF2 LOI of lung cancer foci, matched paracancer tissues, and normal lung tissues were $77.4 \%(24 / 31), 61.3 \%(19 / 31)$, and $29.0 \%(9 / 31)$, respectively. The LOI differences for IGF2 among the three groups were statistically significant $\left(\chi^{2}=15.267, p=0.000\right)$, and the LOI frequency of IGF2 in normal lung tissue was significantly lower than that in lung cancer foci and paracancer tissues $\left(\chi^{2}=14.577, p=0.000 ; \chi^{2}=6.513, p=0.011\right)$. No statistical difference was observed between the lung tumor group and the matched paracancer group $\left(\chi^{2}=1.897, p=0.168\right)$. The prevalence of advanced clinical stages $\left(\chi^{2}=2.379 ; p=0.017\right)$ and lymph node metastasis $\left(\chi^{2}=5.552 ; p=0.018\right)$ was significantly higher for LOIpositive paracancer tissues than for LOI-negative paracancer tissues. Conclusions: IGF2 LOI is highly frequent in Chinese primary lung cancer patients, especially those with increased risk of lymph node metastasis and advanced clinical stages. IGF2 LOI may be an early epigenetic event in human lung carcinogenesis.
\end{abstract}

Keywords: Lung cancer - insulin-like growth factor 2 - loss of imprinting - CENTRAL CHINA

Asian Pac J Cancer Prev, 15 (18), 7799-7803

\section{Introduction}

Genomic imprinting is a non-Mendelian inherited epigenetic form of gene regulation that results in the differential expression of maternal and paternal alleles (Feinberg and Tycko, 2004; Livingstone, 2013). Loss of imprinting (LOI) describes mechanisms that either activate the normally silenced allele or inactivate the normally active gene copy. The IGF2 gene is a maternally imprinted proto-oncogene since a cluster of imprinting genes has been identified in chromosome 11p15.5, thus, the LOI of IGF2 may result in twofold to threefold increase in transcription, which is higher than the theoretical twofold increase (Sakatani et al., 2001; Woodson et al., 2004). Our previous study showed that overexpression of the mitogen IGF2 protein has an important function in the progression of primary lung cancer (Zhang et al., 2009). However, the imprinting status of IGF2 and its LOI incidence in primary lung cancer in Asian patients remains unclear.

To determine whether the LOI of the IGF2 loci is involved in the pathogenesis of primary lung cancer, we examined the LOI status of IGF2 in lung cancer foci, its matched adjacent ( $2 \mathrm{~cm}$ apart) non-tumor tissues, and the most distal tissues from the tumor by using RT-nested PCR-RFLP. We also investigated the association of IGF2 LOI of lung cancer specimens with clinicopathological features.

\section{Materials and Methods}

\section{Patients and tissues}

A total of 216 blocks of fresh tissues were collected from surgical specimens in the Department of Pathology, Tongji Hospital, Huazhong Science and Technology University, between November 2007 and October 2010. Written informed consent was obtained from all patients. All tissues removed surgically were immediately frozen in liquid nitrogen and stored at $-80^{\circ} \mathrm{C}$ until analysis. Samples included lung cancer foci, paracancer tissues ( $2 \mathrm{~cm}$ adjacent from the tumor), and matched normal lung tissues (the most distal tissues from the tumor) as controls from 64 primary lung cancer patients, together with pulmonary benign lesions in 24 noncancer patients. Pulmonary benign lesions consisted of 
tuberculosis, inflammatory pseudotumor, pulmonary fungal granuloma, and bronchiectasia. All the samples were evaluated and confirmed by qualified pathologists through histopathological examination. All subjects were exclusive of other metabolic diseases, such as diabetes mellitus. None of the subjects had received radiation therapy or chemotherapy before surgery.

Screening of heterozygotes for IGF2 gene by PCR-RFLP Nucleic acid preparation and PCR. Genomic DNA (gDNA) was extracted from lung cancer tissue and pulmonary benign lesions, isolated by conventional phenol-chloroform extraction and ethanol precipitation, and dissolved in sterile distilled water. The polymorphic region of the IGF2 gene exon 9 was amplified using the following PCR primers (Wu et al., 1997): 5'-CTT GGA CTT TGA GTC AAA TTG G-3‘ and 5`-GGT CGT GCC AAT TAC ATT TCA-3‘ (Shanghai Bio Asia Biotechnology Co., Ltd). PCR amplification was carried out with an initial denaturation for $5 \mathrm{~min}$ at $94^{\circ} \mathrm{C}$, followed by 30 cycles of denaturation at $94^{\circ} \mathrm{C}$ for $45 \mathrm{~s}$, reannealing at $54^{\circ} \mathrm{C}$ for $45 \mathrm{~s}$, extension at $72^{\circ} \mathrm{C}$ for $45 \mathrm{~s}$, and a final extension of $10 \mathrm{~min}$ at $72^{\circ} \mathrm{C}$.

ApaI RFLP detection. Heterozygosity in the IGF2 gene exon 9 was determined by the presence or absence of ApaI sites in one allele. PCR products from each sample (10 $\mu \mathrm{l})$ were subjected to $10 \mathrm{U}$ of Apa I endonuclease (MBI Fermentas) digestion for $6 \mathrm{~h}$ at $37^{\circ} \mathrm{C}$, and run through $3 \%$ agarose gel electrophoresis stained with ethidium bromide. To visualize the digested fragments, DNA markers (Tiangen, China) were used as molecular weight standards, and agarose gel samples were observed under a UVP-gel imaging analysis system (UVP Ltd, Cambridge, UK).

Because the expected size of the PCR fragment of IGF2 gene is 292 bp, samples exhibiting double bands of 292 and 231 bp were considered heterozygous cases and thus informative for further analysis (Figure 1). Homozygotes were excluded in this work.

\section{Analysis of IGF2 imprinting status by RT-nested PCR- RFLP}

Nucleic acid preparation and RT-nested PCR-RFLP. Total RNA extraction and reverse transcription were prepared from heterozygous specimens including 31 matched lung cancer samples and 9 pulmonary benign lesions using the Superscript II RT Kit (Invitrogen Corporation, USA). The RNA extracts were treated with RNase-free DNase I to eliminate DNA contamination (BRL, Baltimore, MD, USA). Complementary DNA (cDNA) was amplified by first-round PCR using the same primers used for gDNA, and the expected size of the PCR fragment by UVP-gel imaging analysis system was 292 bp. The reaction mixture of the product with the negative PCR fragment $(1 \mu \mathrm{L})$ was subjected to a second round of amplification with inner primers of 5'-CTG GAC TTG AGT CCC TGA AC-3“ and 5‘-CTG GGT CCC TCT GAC TGC TC-3‘ (Shanghai Bio Asia Biotechnology Co., Ltd.). Briefly, the reaction system and cycling conditions were identical to those described above, except that the first-round PCR products were used as the template for second-round PCR. A negative control reaction was prepared in parallel without reverse transcriptase to ensure the elimination of gDNA during cDNA synthesis. The amplified RT-PCR and RT-nested PCR products were then digested with ApaI and electrophoresed on 3.0\% agarose gel as described above, and imaged under UVP to analyze the imprinting status of IGF2.

Evaluation criterion for IGF2 imprinting status. The size of the RT-PCR and RT-nested PCR products were 292 and $198 \mathrm{bp}$, respectively, and their restriction sites for ApaI were located at 231 and 160bp. Thus, the 292 bp/198 bp band in the picture (Figure 2) was named A, whereas the $231 \mathrm{bp} / 160 \mathrm{bp}$ band was designated B (the $61 \mathrm{bp} / 38 \mathrm{bp}$ fragment was too small to be detected). The imprinting status of IGF2 was scored semi-quantitatively using BandScan 5.0 software. According to current international reference standards, the threshold for scoring LOI of IGF2 is defined as a ratio of less than threefold difference in Amax between A and B, otherwise it was considered as normal imprinting of IGF2 (Kohda et al., 2001; Cruz-Correa et al., 2004) (Figure 2).

\section{Statistical analysis}

The prevalence of LOI in patients with lung cancer was described as a proportion. The demographic and clinicopathological characteristics in LOI-positive and LOI-negative patients were compared using the ChiSquare test or Mann-Whitney U non-parameter test where necessary. All $p$ values were two-tailed with a value of 0.05 specified as statistically significant. All data analyses were calculated using SPSS version 15.0 statistical software.

\section{Results}

\section{Heterozygote data}

A total of 31 cases of lung cancer tissues and nine cases of pulmonary benign lesions were identified as heterozygotes with an ApaI transcription polymorphism site (Figure 1). Thirty-one cases of informative lung cancers combined with their matched paracancer and normal lung specimens, as well as nine cases of pulmonary benign lesions, were determined to investigate allelespecific expression of IGF2 gene.

\section{Detection of imprinting status of IGF2 gene heterozygous} cases

Among informative matched lung cancer specimens, $77.4 \%(24 / 31)$ of lung cancer foci, $61.3 \%$ (19/31) of

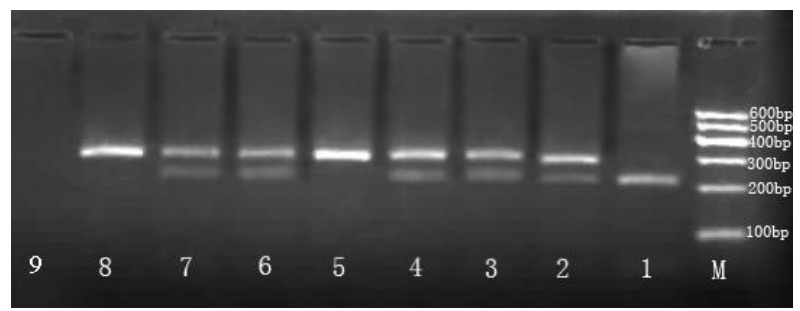

Figure 1. Polymorphism of ApaI Sites in the IGF2 Gene

Exon 9 by PCR-RFLP. M, marker; 1 and 5, homozygotes: 1 displayed both alleles with ApaI sites and 5 displayed both alleles without ApaI sites; 2-4, 6 and 7 yielded double bands of 292 and 231 bp indicating heterozygous specimens; 8, negative control without Apa I endonuclease; 9, blank control 
paracancer specimens, and $29.0 \%(9 / 31)$ of normal lung specimens were found to undergo imprinting loss (Table 1 and Figure 2). Statistical analysis showed that LOI differences for IGF2 among the three groups were statistically significant $\left(\chi^{2}=15.267, p=0.000\right)$, and the LOI of IGF2 between lung cancer foci or paracancer tissues and normal lung tissues showed statistical differences (Table $2 ; \chi^{2}=14.577, p=0.000 ; \chi^{2}=6.513, p=0.011$ respectively). However, no statistically significant differences between the lung cancer and paracancer groups $\left(\chi^{2}=1.897\right.$, $p=0.168)$ were observed. As well, 66.7\% (6/9) of the informative pulmonary benign lesions also showed LOI of IGF2, including one case of tuberculosis, three cases of inflammatory pseudotumor, and two cases of pulmonary fungal granuloma.

\section{Clinicopathological characteristics and IGF2 LOI in the} matched samples

Key clinicopathological parameters associated with IGF2 LOI status in matched lung cancer samples are

Table 1. LOI Analysis of IGF2 in 31 Matched Cases

\begin{tabular}{|c|c|c|c|c|c|}
\hline & LOI $(\%)$ & Normal (\%) & $\chi^{2}$ & $\begin{array}{c}\mathrm{p} \\
\text { value }\end{array}$ & $\begin{array}{l}\text { Coefficient } \\
\text { correlation }\end{array}$ \\
\hline Parameters & & & 15.267 & 0 & 0.398 \\
\hline lung cancer foci & $24(77.4)$ & 7 (22.6) & ${ }^{\triangle} 1.897$ & 0.168 & \\
\hline Paracancer tissue & $19(61.3)$ & $12(38.7)$ & ${ }^{\# \mathbf{\Delta}} 6.513$ & 0.011 & 0.324 \\
\hline Normal lung tissue & $9(29.0)$ & $22(71.0)$ & $* 14.577$ & 0 & 0.485 \\
\hline
\end{tabular}

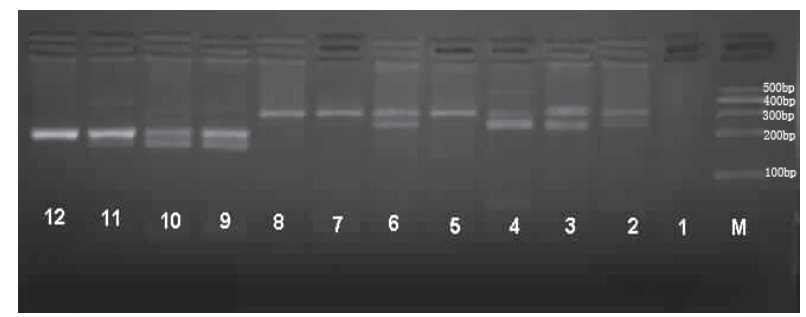

Figure 2. Imprinting Analysis of IGF2 by RT-PCR-

RFLP. M, marker; 1, blank control; 8 and 12, negative controls without Apa I endonuclease; $2-7$, RT-PCR products: 2,3 , and 6 showed expression of both alleles indicating LOI, 4, 5, and 7 showed only one allele expression indicating normal imprinting; 9-11: RT-nested PCR products: 9 and 10 showed both alleles expression indicating LOI, 11 showed only one allele expression indicating normal imprinting summarized in Table 2. Parameters such as age, gender, and smoking history were not statistically correlated with the frequency of IGF2 LOI in the studied specimens (data not shown). The positive rate of IGF2 LOI was significantly higher in paracancer specimens with lymph node metastasis than in those without metastasis $(81.3 \%$ vs $40.0 \% ; p=0.018)$. However, the difference in lung cancer foci or normal lung tissue as controls was not statistically significant. The clinical stage also showed statistical difference with LOI of IGF2 in paracancer specimens $(p=0.017)$ but not in lung cancer foci or normal lung tissues (Table 2).

\section{Discussion}

Lung cancer is a major health problem and the leading cause of cancer deaths worldwide. Given this reason, determining additional etiology and risk factors is very important. The exact function of IGF2 gene polymorphism in human lung cancer cell lines has not yet been reported, and few data are available on the prevalence of LOI of IGF2 in primary lung cancer. In contrast to the findings of Kohda et al., who noted frequent LOI of IGF2 only in lung adenocarcinomas (about 47\%), but not in matched normal tissue (Kohda et al., 2001), the present study showed that $77.4 \%$ of lung cancers as well as $29.0 \%$ of matched normal lung tissues displayed LOI of IGF2. In addition, the difference of LOI of IGF2 between lung cancers and normal tissues was statistically different. However, LOI was not always found in the normal tissues in some cancers, Woodson et al. assessed that LOI of IGF-2 may be a tumor-specific event and a predisposing factor only for certain cancer types (Woodson et al., 2004). In the present study, six cases of pulmonary benign lesions exhibited LOI of IGF2, which indicates that the mitogenic actions of IGF2 protein are essential to cell proliferation in benign proliferative diseases. Subsequent histopathological examination confirmed that six cases of benign diseases showed a large number of proliferative fibroblasts and infiltrating lymphocytes, which, to some extent, result in potential false-positive results (Kohda et al., 2001). These results are consistent with the function of IGF2 protein as a growth-promoting factor. Overall, a high frequency of IGF2 LOI was present in patients with lung cancer in the Central China region. These findings indicate that LOI of IGF2 in the lung may predispose individuals to develop lung cancer and that LOI of IGF2 may be an epigenetic trait that is polymorphic in the

Table 2. Relationship between IGF2 Imprinting Status and key Clinicopathological Characteristics in Matched Lung Cancer Samples

\begin{tabular}{|c|c|c|c|c|c|c|c|c|c|c|}
\hline \multirow[t]{2}{*}{ Parameters } & & \multicolumn{3}{|c|}{ Lung cancer foci } & \multicolumn{3}{|c|}{ Paracancer tissue } & \multicolumn{3}{|c|}{ Normal lung tissue } \\
\hline & & $\mathrm{LOI}(+)$ & LOI(-) & $p$ value & $\mathrm{LOI}(+)$ & LOI(-) & $p$ value & $\mathrm{LOI}(+)$ & LOI(-) & $p$ value \\
\hline \multirow[t]{2}{*}{ Lymph node } & Metastasis & 12 & 4 & \multirow[t]{2}{*}{0.739} & 13 & 3 & \multirow[t]{2}{*}{0.018} & 6 & 10 & \multirow[t]{2}{*}{0.499} \\
\hline & Non-metastasis & 12 & 3 & & 6 & 9 & & 3 & 12 & \\
\hline \multirow[t]{2}{*}{ Histological type } & $\mathrm{SCC}$ & 9 & 3 & \multirow[t]{2}{*}{1} & 8 & 4 & \multirow[t]{2}{*}{0.717} & 1 & 11 & \multirow[t]{2}{*}{0.101} \\
\hline & $\mathrm{ACC}$ & 15 & 4 & & 11 & 8 & & 8 & 11 & \\
\hline \multirow[t]{3}{*}{ Stage } & I & 8 & 2 & \multirow[t]{3}{*}{0.957} & 3 & 7 & \multirow[t]{3}{*}{0.017} & 1 & 9 & \multirow[t]{3}{*}{0.124} \\
\hline & II & 2 & 1 & & 2 & 1 & & 1 & 2 & \\
\hline & III $\sim$ IV & 14 & 4 & & 14 & 4 & & 7 & 11 & \\
\hline
\end{tabular}

*SCC: squamous cell carcinoma; ACC: adenocarcinoma 
population. Cui et al. reported that LOI of IGF2 may provide a marker for identifying an important subset of the population with cancer or those at risk of developing cancer (Cui et al., 1998). To improve the survival rate of patients with lung cancer, innovative treatment based on a specific molecular target is necessary. Gene therapy targeting the loss of the IGF2 imprinting system via apoptosis induction in vitro and in vivo in human cancer cells was initially carried out (Pan et al., 2010; Nie et al., 2012). Although more investigations are required for a wide range of clinical applications, our present findings support this novel approach.

IGF2 protein is an autocrine, paracrine, and endocrine growth factor, and the expression of this protein is regulated in a development-dependent and tissue-specific manner. To our knowledge, the exact mechanism of action of IGF2 in human cancers has not been established (Linnerth et al., 2005; Vu et al., 2010; Brouwer-Visser et al., 2014; Yang et al., 2014). In contrast to earlier report, our findings suggested that high-frequency LOI of IGF2 is statistically associated with lung cancer foci and paracancer tissues, but not with matched normal lung tissues. Taken together, our studies and Kaneda et al.'s previous report (Kaneda et al., 2007) provide new insights on the function of IGF2 LOI, indicating that the LOI of IGF2 is most likely to be an early epigenetic event in human lung carcinogenesis. Therefore, the biological effect of IGF2 LOI can facilitate the proliferation of tumorigenic cells at early cancer stages. The expression of IGF2 protein is most likely supported by autochthonous LOI of tumor cells and neighboring cells around the tumor. Thus, large amounts of secreted IGF2 protein may interact with the IGF1R of its own cells as well as around cancer cells and, in turn, accelerate the mitogenic activity of these cancer cells. Nosho et al. reported that the tumor cells overproduce IGF2 protein, which in turn bind to and activate the IGF1R, on the same tumor cells by an autocrine loop mechanism in the early stage of colorectal carcinogenesis (Nosho et al., 2004). This phenomenon partly explains our findings. Although the mechanisms for initiating and maintaining imprinting or LOI of IGF2 are not clearly elucidated, we hypothesized that IGF2 appears to exert autocrine/paracrine effects on growth regulation in human lung cancer rather than an endocrine effect. Moreover, Fumio Sakauchi et al. reported that serum IGF2 was not associated with the breast cancer risk in postmenopausal Japanese women (Sakauchi et al., 2009), however, it was negatively associated with the breast cancer risk in premenopausal women. Thus, tissuespecific levels of IGF2 protein are more relevant than circulatory serum IGF2 protein levels in the maintenance of human cancers. Further studies are needed to support these findings.

We previously showed that overexpression of mitogen IGF2 protein exhibited important function in the progression of primary lung cancer and is related to lymph node metastasis, differentiation, and clinical stage (Zhang et al., 2009). However, limited data on the association between IGF2 LOI and clinical characteristics in lung cancer are available. Kohda et al. showed that LOI of IGF2 is observed frequently in poorly differentiated adenocarcinomas (Kondo et al., 1996). In the present study, clinical characteristics (lymph node metastasis and clinical stage) were independently associated with the LOI of IGF2 in paracancer tissues $(2 \mathrm{~cm}$ distant from the tumor) but not with the LOI of IGF2 in lung cancer foci or normal lung tissues. We found that patients with LOI of IGF2 in their paracancer tissues had increased risk of lymph node metastasis compared with those without $(p=0.018)$. Therefore, we speculate that IGF2 promotes lymph node metastasis mainly by a paracrine mechanism. In addition, the association of IGF2 LOI with lymph node metastasis contributes to the development and progression of lung cancer. IGF2 LOI is reported to be associated with epigenetic modification (hypermethylation or hypomethylation) of relevant sites in differentially methylated region of the IGF2/H19, allowing activation of the normally silent maternal allele of IGF2 (Tian et al., 2012; Gao et al., 2014). Although these findings are interesting, they must be confirmed in larger studies. Furthermore, identification of patients at high risk of lymph node metastasis is necessary because they should be supported by more active radiotherapy and chemotherapy.

In conclusion, to our knowledge, this study is the first to demonstrate the prevalence of IGF2 gene polymorphism and the LOI of IGF2 in patients with primary lung cancer in the English literature. We demonstrated that a high frequency of primary lung cancer patients in the Central China region had IGF2 LOI, which is involved in the early stage of primary lung cancer with potential clinical application for early diagnosis and screening. Moreover, the association of IGF2 LOI with lymph node metastasis may contribute to the development and progression of primary lung cancer. Additional large-scale studies elucidating the exact function of IGF2 imprinting changes in lung tumorigenesis in different ethnic populations are warranted.

\section{Acknowledgements}

This work was supported by grants from National Natural Science Foundation of China (contract No. 30400358).

\section{References}

Brouwer-Visser J, Lee J, McCullagh K, et al (2014). Insulin-like growth factor 2 silencing restores taxol sensitivity in drug resistant ovarian cancer. PLoS One, 9, 100165.

Cui H, Horon IL, Ohlsson R, et al (1998). Loss of imprinting in normal tissue of colorectal cancer patients with microsatellite instability. Nat Med, 4, 1276-80.

Cruz-Correa M, Cui H, Giardiello FM, et al (2004). Loss of imprinting of insulin growth factor II gene: a potential heritable biomarker for colon neoplasia predisposition. Gastroenterology, 126, 964-70.

Feinberg AP, Tycko B (2004). The history of cancer epigenetics. Nat Rev Cancer, 4, 143-53.

Gao T, He B, Pan Y, et al (2014). H19 DMR methylation correlates to the progression of esophageal squamous cell carcinoma through IGF2 imprinting pathway. Clin Transl Oncol, 16, 410-7.

Kaneda A, Wang CJ, Cheong R, et al (2007). Enhanced sensitivity to IGF-II signaling links loss of imprinting of 
IGF2 to increased cell proliferation and tumor risk. Proc Natl Acad Sci USA, 104, 20926-31.

Kohda M, Hoshiya H, Katoh M, et al (2001). Frequent loss of imprinting of IGF2 and MEST in lung adenocarcinoma. Mol Carcinog, 31, 184-91.

Kondo M, Takahashi T (1996). Altered genomic imprinting in the IGF2 and H19 genes in human lung cancer. Nihon Rinsho, 54, 492-6.

Linnerth NM, Baldwin M, Campbell C, et al (2005). IGF-II induces CREB phosphorylation and cell survival in human lung cancer cells. Oncogene, 24, 7310-9.

Livingstone C (2013). IGF2 and cancer. Endocr Relat Cancer, 20, 321-39.

Nie ZL, Pan YQ, He BS, et al (2012). Gene therapy for colorectal cancer by an oncolytic adenovirus that targets loss of the insulin-like growth factor 2 imprinting system. Mol Cancer, 11, 86.

Nosho K, Yamamoto H, Taniguchi H, et al (2004). Interplay of insulin-like growth factor-II, insulin-like growth factor-I, insulin-like growth factor-I receptor, COX-2, and matrix metalloproteinase-7, play key roles in the early stage of colorectal carcinogenesis. Clin Cancer Res, 10, 7950-7.

Pan Y, He B, Li T, et al (2010). Targeted tumor gene therapy based on loss of IGF2 imprinting. Cancer Biol Ther, 10, 290-8.

Sakauchi F, Nojima M, Mori M, et al (2009). Serum insulin-like growth factors I and II, insulin-like growth factor binding protein-3 and risk of breast cancer in the Japan Collaborative Cohort study. Asian Pac J Cancer Prev, 10, 51-5.

Sakatani T, Wei M, Katoh M, et al (2001). Epigenetic heterogeneity at imprinted loci in normal populations. Biochem Biophys Res Commun, 283, 1124-30.

Tian F, Tang Z, Song G, et al (2012). Loss of imprinting of IGF2 correlates with hypomethylation of the H19 differentially methylated region in the tumor tissue of colorectal cancer patients. Mol Med Rep, 5, 1536-40.

$\mathrm{Vu}$ TH, Nguyen AH, Hoffman AR (2010). Loss of IGF2 imprinting is associated with abrogation of long-range intrachromosomal interactions in human cancer cells. Hum Mol Genet, 19, 901-19.

Woodson K, Flood A, Green L, et al (2004). Loss of insulin-like growth factor-II imprinting and the presence of screendetected colorectal adenomas in women. $J$ Natl Cancer Inst, 96, 407-10.

Wu HK, Squire JA, Song Q, et al (1997). Promoter-dependent tissue-specific expressive nature of imprinting gene, insulinlike growth factor II, in human tissues. Biochem Biophys Res Commun, 233, 221-6.

Woodson K, Flood A, Green L, et al (2004). Loss of Insulin-Like Growth Factor-II Imprinting and the Presence of ScreenDetected Colorectal Adenomas in Women. J Natl Cancer Inst, 96, 407-10.

Yang B, Wagner J, Damaschke N, et al (2014). A novel pathway links oxidative stress to loss of insulin growth factor-2 (IGF2) imprinting through NF- $x \mathrm{~B}$ activation. PLoS One, 18, 88052.

Zhang M, Wu CH, Wang YJ, et al (2009). The relationship between HPV infection and the expression of protein insulin-like growth factor II in lung cancer and their clinical significance. Tumor, 29, 749-53. 